\title{
¿QUÉ ES UN MOVIMIENTO SOCIAL FUERTE? CONCEPTUALIZACIONES DE LA NOCIÓN DE FORTALEZA Y APLICACIÓN AL CASO DEL MOVIMIENTO ESTUDIANTIL 2002-2011 EN CHILE
}

\author{
CARMEN SILVA DREYER \\ JAVIER ROMERO OCAMPO**
}

\begin{abstract}
RESUMEN
Los movimientos sociales persiguen propósitos colectivos en interacción con oponentes. Su poder de influencia dependerá de su nivel de fortaleza. El presente artículo ofrece una nueva síntesis conceptual de la noción de fortaleza a partir de las descripciones presentes en la literatura sobre movimientos sociales y los elementos del concepto de fortalecimiento o empoderamiento provenientes de la psicología comunitaria. Mediante esta herramienta conceptual se analiza teóricamente el movimiento estudiantil entre los años 2002 y 2011. Se deduce que se trata de un movimiento con importantes fortalezas, como sentidas demandas, un consensuado marco interpretativo en lo esencial y logro de simpatía en la población, una importante motivación de control, una organización buena y horizontal, y tácticas directas. En cambio, merece duda el nivel de logro de metas, la identidad como movimiento, la claridad de fronteras internas y externas, y las oportunidades políticas aprovechables a su favor.
\end{abstract}

\section{PALABRAS CLAVE: MOVIMIENTO ESTUDIANTIL, FORTALEZA, EMPODERAMIENTO}

\footnotetext{
* Psicóloga, magíster en psicología social comunitaria, investigadora Universidad Alberto Hurtado, Santiago, Chile.

E-Mail: casilvdr@uahurtado.cl.

** Profesor de historia y geografía, sociólogo y psicólogo, doctor en estudios americanos, mención pensamiento y cultura, Universidad de Santiago de Chile. Académico Escuela de Psicología, Universidad Central de Chile. E-Mail: jromeroo@ucentral.cl.

Este artículo forma parte de una investigación en curso sobre el movimiento estudiantil actual, financiada por la Universidad Alberto Hurtado.
} 


\title{
O QUE É UM MOVIMENTO SOCIAL FORTE? A CONCEITUAÇÃO DA NOÇÃO DE FORÇA E APLICAÇÃ̃O AO CASO DO MOVIMENTO ESTUDANTIL 2002-2011 NO CHILE
}

\begin{abstract}
RESUMO
Os movimentos sociais buscam objetivos coletivos em negociação com adversários. Seu poder de influência dependerá do seu nível de força. Este artigo oferece uma nova síntese conceitual da noção de força a partir das descrições presentes na literatura sobre movimentos sociais e os elementos do conceito de fortalecimento ou empoderamento da psicologia comunitária. Por meio desta ferramenta conceitual, analisa-se teoricamente o movimento estudantil entre os anos de 2002 e 2011. Conclui-se que o movimento possui pontos fortes, tais como: demandas legítimas, um marco interpretativo consensual em sua essência, a simpatia da população, uma importante motivação de controle, boa organização horizontal e táticas diretas. Por outro lado, devem ser questionados o nível de cumprimento das metas, a identidade como um movimento, a clareza das fronteiras internas e externas e as oportunidades políticas que beneficiam o movimento.
\end{abstract}

PALAVRAS CHAVE: MOVIMENTO ESTUDANTIL, FORÇA, EMPODERAMENTO

\section{WHAT IS A STRONG SOCIAL MOVEMENT? CONCEPTUALIZATION OF THE CONCEPT OF STRENGTH AND APPLICATION TO THE CASE OF THE STUDENT MOVEMENT IN CHILE 2002-2012}

\begin{abstract}
Social movements pursue collective goals in interaction with opponents. Its power of influence will depend of their level of strength. This article offers a new conceptual synthesis of the notion of strength from descriptions in literature about social movements and the elements of the concept of strengthening or empowerment from community psychology. Through this conceptual tool is analyzed theoretically the student movement between the years 2002 and 2011. It follows that it is a movement with important strengths, as heartfelt demands, a consensual interpretative framework in the essential and achievement of sympathy in the population, a major motivation of control, a good horizontal organization and direct tactics. On the other hand, deserves no doubt the level of attainment of goals, the identity as a movement, the clarity of internal and external borders, and exploitable opportunities policies in their favor.
\end{abstract}

KEY WORDS: STUDENT MOVEMENT, STRENGTH, EMPOWERMENT 


\section{INTRODUCCIÓN}

LOS MOVIMIENTOS SOCIALES (MS) constituyen expresiones de la vida democrática. Se han definido como desafíos colectivos basados en propósitos comunes y solidaridades sociales —en interacción sostenida con élites, oponentes y autoridades - en disputa con agentes de poder (Tarrow, 1998, citado en Meyer y Verduzco, 2010). Su poder de influencia dependerá de su nivel de fortaleza, por lo que la construcción de ella es básica para su existencia y la comprensión de lo que constituye es indispensable para un análisis del fenómeno. En consecuencia: ¿Qué es la fortaleza en un movimiento social? ¿Qué herramientas conceptuales tenemos para analizarla en movimientos concretos? ¿Qué tan fuerte es uno de los movimientos que ha destacado en la última década, cual es, el movimiento estudiantil (ME)?

En el presente artículo pretendemos ofrecer una nueva síntesis conceptual de la noción de «fortaleza» en los MS, basada tanto en caracterizaciones de fortaleza presentes en la literatura sobre el movimiento social, como en los elementos del constructo de empoderamiento/fortalecimiento de la psicología comunitaria. Luego utilizaremos esta herramienta conceptual para desarrollar un diagnóstico teórico sobre la fortaleza de un movimiento social concreto posterior al año 2000, el cual es el movimiento estudiantil en Chile, a partir de antecedentes bibliográficos existentes.

\section{CONCEPTUALIZACIONES DE FORTALEZA EN LOS MS}

La fortaleza de un movimiento social se ha definido acorde a distintos factores. Incluye una gran identificación de sus participantes con la demanda y el marco interpretativo de ésta, y la necesidad muy sentida o imperiosa de participar para generar cambios de sus situaciones (Klandermans, 2003; Meyer y Verduzco, 2010). También comprende percibir injusticias, indignación moral, dar sentido a la existencia y expresar creencias (Klandermans, 2003). Asimismo, encierra el querer actuar como miembros de un grupo con una identidad social (Klandermans, 2003; Meyer y Verduzco, 2010). La fortaleza a su vez se asocia a la efectividad y el logro de metas a costos razonables e incentivos directos asociados (Falero, 2007; Klandermans, 2003; Meyer y Verduzco, 2010; Olson, 1992). Además, en la fortaleza inciden aspectos organizativos y recursos: la capacidad de liderazgo atento, estratégico y desinteresado, la habilidad de organización y vinculación en 
redes, la disponibilidad y flujo de recursos de dinero, tiempo, esfuerzo, el repertorio de estrategias que apelan a la gente y que se ajustan a «oportunidades políticas, recursos, valores e identidades», y la capacidad de innovar (Klandermans, 2003; Meyer y Verduzco, 2010). La fortaleza también implica la participación masiva en las convocatorias (Kirshner, 2006). Asimismo, es importante el interés de la sociedad en las metas del movimiento (Klandermans, 2003). Al respecto, Granovetter (1985, citado en Falero, 2007) destaca el concepto de embeddedness, que significa que la acción social no está separada de la búsqueda de aprobación social, del estatus, de la sociabilidad y del poder. En relación con la institucionalidad, influyen las oportunidades políticas que permitan mayor impacto, y la capacidad del movimiento de eliminar barreras a la participación (Meyer y Verduzco, 2010).

La fortaleza también puede ser entendida a partir de los siguientes factores contenidos en el texto de Meyer y Verduzco (2010), basado en una revisión de la literatura sobre movimientos sociales: (a) Razones y surgimiento de un movimiento con sentidos, motivos o demandas instrumentales, creencia de efectividad, necesidad sentida de hacerlo para generar cambios, identificación con un marco interpretativo de las demandas, y oportunidades (circunstancias) políticas externas como políticas públicas amenazadoras o por el contario, prometedoras; (b) Identidad social que permite delinear las fronteras del movimiento, con sus lazos internos y clara definición de oponentes. A ello también contribuye su marco interpretativo; (c) Disponibilidad y flujo de recursos de dinero, tiempo, esfuerzo; buena organización y repertorio de estrategias ajustadas a «oportunidades políticas, recursos, valores e identidades», y capacidad de innovar; satisfacción de demandas antitéticas al interior del mismo movimiento; liderazgos que no buscan sus ganancias políticas ( $v . g$. cultivar relaciones con autoridades y frenar acciones disruptivas) y que estén atentos a las circunstancias políticas, a los cambios en la prominencia de tópicos y alineamiento con aliados. Estos factores están interrelacionados en un proceso dinámico y cambiante.

\section{CONCEPTUALIZACIONES DE EMPODERAMIENTO}

Los conceptos de fortaleza descritos anteriormente y el constructo de empoderamiento tienen bastante semejanza entre sí, tanto en el término como en su significado. Al respecto, Montero (2003) aboga por el uso de la palabra «fortalecimiento» en vez de «empoderamiento». El 
fortalecimiento ha sido definido como «el proceso mediante el cual los miembros de una comunidad (individuos interesados y grupos organizados) desarrollan conjuntamente capacidades y recursos para controlar su situación de vida, actuando de manera comprometida, consciente y crítica, para lograr la transformación de su entorno según sus necesidades y aspiraciones, transformándose al mismo tiempo a sí mismos» (Montero, 2003). Entre los elementos fundamentales que forman parte del empoderamiento menciona: La participación en función de objetivos surgidos de necesidades, en presencia de solidaridad; la conciencia o desarrollo de la crítica y comprensión del carácter histórico de los fenómenos comunitarios; el control de las personas en sus comunidades sobre circunstancias del orden social y recursos y; el poder social de la comunidad; la autogestión de las acciones y decisiones de la comunidad y autoeficiencia en la organización comunitaria; compromiso, entendido como un sentimiento de apego y obligación para con la comunidad y participación en acciones que la beneficien; desarrollo y expresión de capacidades individuales, competencias de distinto tipo de los miembros de una comunidad. dentidad social comunitaria con sus valores y creencias. Por su parte, Zimmerman (2000) plantea un modelo de empoderamiento psicológico constitutivo de tres componentes, que son: i) intrapersonal, que comprende la motivación de control, percepción de control y autoeficacia); ii) interaccional, que engloba destrezas para interactuar con el ambiente para lograr control (v.g. toma de decisiones), movilización de recursos, habilidades analíticas de la situación sociopolítica en cuanto a conciencia de las oportunidades existentes para lograr metas y de agentes causales de problemas; iii) comportamental, entendida como el despliegue de acciones para lograr las metas. Definió el empoderamiento como creencias en la propia competencia, esfuerzos para ejercer control y una comprensión crítica de la realidad sociopolítica.

\section{DEFINICIÓN INTEGRADORA DE FORTALEZA/EMPODERAMIENTO}

A partir de las semejanzas - y también las particularidades que aporta cada concepto - podemos construir una descripción integradora de elementos de empoderamiento/fortaleza, pertinente en este caso para movimientos sociales en general, y para el movimiento estudiantil chileno en particular. Esta definición se basa en los autores Klandermans (2003), Meyer y Verduzco (2010), Zimmerman (2000), Montero (2003) y Rappaport (1981). Los elementos de fortaleza son dinámicos, 
son vividos como proceso y se interrelacionan entre sí. Ellos se resumen en los siguientes aspectos: i) el objetivo de transformación social, según sus necesidades, expresadas como demandas y el ejercicio del análisis sociopolítico de la realidad que permite la construcción de un marco interpretativo en el que las demandas se insertan, se justifican, y el problema se explica. Ello implica una identificación con una demanda (instrumental, ideológica, identitaria) y con el marco interpretativo. También, una percepción subjetiva de apoyo recibido del exterior respecto de esas demandas e interpretación de la realidad («movilización de consenso») y una percepción de ser valorados por personas significativas simpatizantes; ii) sentido de control e influencia: efectividad en el logro de las metas del movimiento, sentido de autoeficacia y creencia en la propia competencia. También, percepción de control, sentido de agencia, motivación de control («querer cambiar sus situaciones») y creencia en la necesidad imperiosa de participar para generar cambios - lo que se asocia a demandas percibidas como muy relevantes (y conexión de esto con sus opciones de vida o proyectos biográficos) - . A su vez adquiere importancia la evaluación de costos/incentivos de la participación; iii) existencia de sellos identitarios, identificación con el movimiento en general, e implicancias positivas para las identidades individuales y sociales, fronteras con sus lazos internos y definición de oponentes; iv) organización, estrategias y acciones: adecuada organización comunitaria y sentido de autonomía de las acciones y decisiones de la comunidad. Ello incluye la capacidad para conciliar diferencias al interior del movimiento. También, la participación efectiva en la organización y la toma de decisiones. Asimismo, comprende la expresión libre, el ser escuchado y escuchar. A su vez, engloba la identificación con las acciones (por sus valores e identidades) y participación en éstas, los espacios de creatividad e innovación. También incluye la dedicación de recursos propios y la búsqueda de recursos. Finalmente, comprende la toma de opción de involucramiento cívico, el compromiso, la percepción de contar con líderes desinteresados y competentes; v) existencia de oportunidades políticas (circunstancias, contextos) externas como políticas públicas amenazadoras o por el contario, prometedoras y afrontamiento a la institucionalidad.

Por último, cabe señalar que el empoderamiento no se limita al nivel individual, sino que incluye el nivel colectivo que refiere a grupos, organizaciones, entre otros agregados sociales (Silva y Martínez, 2004; Zimmerman, 2000), por lo que es aplicable a los movimientos sociales. 


\section{TABLA RESUMEN. DEFINICIÓN INTEGRADORA DE FORTALEZA A PARTIR DE NOCIONES DE FORTALEZA DE UN MOVIMIENTO SOCIAL Y EL CONCEPTO DE «EMPODERAMIENTO».}

\begin{tabular}{|c|c|c|}
\hline FORTALEZA EN MS & $\begin{array}{l}\text { EMPODERAMIENTO/ } \\
\text { FORTALECIMIENTO }\end{array}$ & $\begin{array}{c}\text { DEFINICIÓN INTEGRADORA } \\
\text { DE FORTALEZA }\end{array}$ \\
\hline $\begin{array}{l}\text { Identificación con la } \\
\text { demanda que persigue } \\
\text { cambios sociales } \\
\text { Construcción de un } \\
\text { marco interpretativo } \\
\text { (interpretación crítica } \\
\text { de la realidad) } \\
\text { Interés de la sociedad } \\
\text { en las metas del mo- } \\
\text { vimiento (moviliza- } \\
\text { ción de consenso) }\end{array}$ & $\begin{array}{l}\text { El empoderamiento persi- } \\
\text { gue que personas, comuni- } \\
\text { dades logren control sobre } \\
\text { sus situaciones de vida y } \\
\text { transformación de su entor- } \\
\text { no según sus necesidades } \\
\text { Habilidades interaccionales } \\
\text { analíticas de comprensión } \\
\text { crítica de la realidad }\end{array}$ & $\begin{array}{l}\text { El objetivo de transfor- } \\
\text { mación social según } \\
\text { necesidades/demandas } \\
\text { Marco interpretativo } \\
\text { Análisis sociopolítico de } \\
\text { la realidad }\end{array}$ \\
\hline $\begin{array}{l}\text { Necesidad sentida de } \\
\text { participar para gene- } \\
\text { rar cambios } \\
\text { Efectividad y logro de } \\
\text { metas a costos razo- } \\
\text { nables e incentivos } \\
\text { directos asociados }\end{array}$ & $\begin{array}{l}\text { Motivación de control } \\
\text { Percepción de control y } \\
\text { autoeficacia } \\
\text { Lograr control sobre orden } \\
\text { social y recursos, poder } \\
\text { social }\end{array}$ & $\begin{array}{l}\text { Sentido de control e } \\
\text { influencia }\end{array}$ \\
\hline $\begin{array}{l}\text { Querer actuar como } \\
\text { miembros de un grupo } \\
\text { con una identidad }\end{array}$ & Identidad social & $\begin{array}{l}\text { Existencia de sellos iden- } \\
\text { titarios }\end{array}$ \\
\hline $\begin{array}{l}\text { Aspectos organizati- } \\
\text { vos, estrategias, re- } \\
\text { cursos }\end{array}$ & $\begin{array}{l}\text { Desarrollar conjuntamente } \\
\text { capacidades y recursos } \\
\text { Autogestión } \\
\text { Habilidades interaccionales } \\
\text { (organización, toma de } \\
\text { decisiones, negociación, } \\
\text { etcétera) } \\
\text { Participar } \\
\text { Componente comportamen- } \\
\text { tal }\end{array}$ & $\begin{array}{l}\text { Organización, estrategias } \\
\text { y acciones }\end{array}$ \\
\hline $\begin{array}{l}\text { Oportunidades políti- } \\
\text { cas }\end{array}$ & Contexto empoderador & $\begin{array}{l}\text { Existencia de oportunida- } \\
\text { des políticas del contexto }\end{array}$ \\
\hline
\end{tabular}

Fuente: elaboración propia. 


\section{ANÁLISIS DE LA FORTALEZA/EMPODERAMIENTO EN EL MS 2002-2011}

En cuanto al objetivo de «transformación social expresado en las demandas», el movimiento estudiantil ha impulsado claras demandas (Huidobro, 2011) que son de tipo instrumental y que refieren en síntesis a un mejoramiento de la equidad en la calidad de la educación y en el acceso a todos sus niveles (Huidobro, 2011). Osorio (2003:11) destaca «el derecho a una educación digna, gratuita, integral y para todos». Algunas demandas más específicas han girado en torno al arancel del pasaje escolar y en el pago de la Prueba de Selección Universitaria (PSU) (Osorio, 2003). Las demandas son profundas, ya que están basadas en una alta insatisfacción de parte de los jóvenes. Según los estudios de sobre creencias juveniles de ciudadanía de Martínez, Silva y Hernández (2010), y Martínez, Silva, Carmona y Cumsille (2012), dicha insatisfacción se hace evidente entre los jóvenes debido a que perciben la vulneración de derechos sociales y la acentuada inequidad social en Chile. En estos estudios se revela que los jóvenes participantes tienen un juicio negativo en relación al estado actual de la educación, la salud, la seguridad ciudadana y las oportunidades en general. Los jóvenes critican la hegemonía del dinero para acceder a oportunidades y lograr superación social, la elevada segregación territorial de sectores sociales, las marcadas clases sociales y elevados niveles de discriminación social, y la atribución de superioridad de aquellos que tienen mayor poder económico, entre otros. Aspiran a la equidad en educación y otros servicios sociales. En concordancia con estas apreciaciones, Contreras, Corrales y Sandoval (1996) encontraron que jóvenes en desventaja social evalúan negativamente la funcionalidad de las oportunidades existentes para la juventud de sectores socioeconómicos bajos, no conducentes a una mejor calidad de vida. Todas estas creencias y «sentires» constituyen el trasfondo de las demandas.

Basado en Pinquart y Silbereisen (2004) y Bronfenbrenner y Ceci (1994), las creencias anteriores también se pueden interpretar a la luz de la tarea del desarrollo evolutivo de la etapa vital juvenil. En la adolescencia «el sí mismo», o «self», evalúa y selecciona opciones disponibles de cara a sus metas, desarrolla estrategias para lograrlas (coping) y obtiene resultados. Las demandas del movimiento estudiantil sugieren que los y las jóvenes están realizando una evaluación negativa de oportunidades de roles disponibles presentes (en sus colegios, en los territorios en los que viven) y futuros (observación del mundo 
adulto, su trabajo y cansancio, malestar) y una evaluación deficitaria de la funcionalidad de los roles (que «sirvan», que conduzcan a una calidad de vida). También están acusando una amenaza para la identidad en términos de poder ser víctimas de una valoración social negativa que encierra discriminación y posición social de inferioridad. La valoración social negativa produce a su vez falta de sentido de pertenencia con sus colegios desprestigiados y discriminados. Este escenario se constituye en un peligro para la identidad e inserción social exitosa.

Según la teoría de los movimientos sociales, las demandas se ligan al ejercicio de un análisis crítico de la realidad sociopolítica y una construcción de un marco interpretativo de la demanda. El marco interpretativo del movimiento en la última década contiene en términos generales, una visión de la educación como derecho ciudadano en contraposición a una visión de educación como bien de consumo. Se concibe así al «estudiante» como sujeto de derechos que han sido violentados, y cuyo garante debe ser el Estado, que en este caso no los protege y no asume su rol (Confederación de Estudiantes de Chile, Confech, 2011; Osorio, 2003). Postulamos que este marco interpretativo debería identificar masivamente a los jóvenes, ya que estudios anteriores (Martínez, Silva, Carmona y Cumsille, 2012; Martínez, Silva y Hernández, 2010) demuestran la inconformidad con el «dinero restrictor de oportunidades» y dificultades de acceso a servicios sociales «comprados» (Silva y Silva, 2010:39-50). Y a la luz de estos estudios, los jóvenes están evaluando, con diferentes niveles de elaboración, el estado de la ciudadanía actual (Martínez, Silva, Carmona y Cumsille, 2012; Martínez, Silva y Hernández, 2010). El marco también logra un vasto apoyo o una amplia «movilización de consenso» de la población chilena, con un respaldo de aproximadamente el setenta y nueve por ciento de los chilenos (Encuesta Adimark, septiembre 2011). Desde estos puntos de vista, el ME cuenta con un marco que da fortaleza y legitimidad a su actuar. Está por verse si este marco es suficiente para afrontar al marco ideológico institucional hegemónico, o si requiere de una mayor elaboración.

En cuanto al sentido de control e influencia, se dispone de pocas evidencias empíricas. Las existentes apuntan a que sus acciones buscan efectividad a corto plazo, con metas palpables (Baeza y Sandoval, 2009). También, que tienen sentimientos ambiguos en relación a la efectividad de las movilizaciones para lograr cambios (Martínez, Silva, Carmona y Cumsille, 2012). Su empoderamiento es relativo en 
este sentido, faltan mayores evidencias. Lo que sí llama la atención es la elevada motivación por participar y por lograr esos cambios deseados, si consideramos como indicador la duración del ME de al menos seis meses consecutivos con presencia de movilizaciones, paros y «tomas» a nivel nacional (Salazar, 2011). La motivación de control constituye un componente de empoderamiento intrapersonal (Zimmerman, 2000), que en este caso es acentuado.

Otro aspecto del empoderamiento/fortaleza en los movimientos se refiere a la posesión de una identidad social. Según los teóricos de la identidad social, ésta implica un proceso de categorización como grupo, diferenciación de otros grupos, evaluación de la posición del grupo en comparación a otros e identificación afectiva o pertenencia al grupo. Al respecto, no se ha estudiado el movimiento estudiantil estrictamente en relación a esos elementos, sino que desde una visión de la identidad grupal entendida en sentido más amplio. Se ha documentado que la identidad colectiva se produce con la propia organización con su cultura y símbolos, observándose una multiplicidad de identidades debido a la participación de los y las jóvenes en diversas organizaciones (Osorio, 2003), más que con el movimiento en su conjunto. El estudio de Muñoz (2002), sobre movimiento juvenil y cultura, coincide con Osorio (2003) en cuanto a la importancia del componente cultural, que ya se observa desde la dictadura militar en adelante y que hoy en día es un componente claro del movimiento estudiantil (Feixa, Saura y Costa 2002; Zarzuri, 2006; ambos citados en Baeza y Sandoval, 2009). Se observa la «carnavalización del poder» que incluye bailes, música, actuaciones y acción de creación de conciencia sobre el problema por medio de folletos y otros medios (Osorio, 2003), lo que puede estar relacionado con un sentido de identidad. Sus acciones tienen efectos simbólicos importantes y de alta originalidad (Baeza y Sandoval, 2009). Han habido una presencia parcial de elementos identitarios distintivos comunes al movimiento estudiantil en su conjunto. Por ejemplo, el movimiento estudiantil secundario del año 2006 en nuestro país tenía la simbología común del pingüino, reflejado en sus pancartas, lienzos, murales, chapitas, entre otros, que pudiera estar reflejando un proceso de categorización grupal y de pertenencia afectiva. Pero el movimiento del año 2011, compuesto por secundarios y universitarios, no tiene aquello. En algunos movimientos del mundo, la identidad social ha sido determinante para la participación, como lo demuestra un estudio que verifica el rol causal de la categorizaciónsocial versus el análisis de costo-beneficio para involucrarse. Es el 
caso de un movimiento de adultos mayores en Alemania denominado gray panters (Simon et al., 1998). Por lo tanto, no queda claro el tema de la identidad social en el ME: ¿existe una identificación con el grupo, más allá del hecho de ser estudiantes, como la tienen, por ejemplo, los campesinos ${ }^{1}$ holandeses por ser parte de su movimiento? ¿Les confiere una «marca» como generación? ¿Se evalúan positivamente como grupo en comparación con otros? Tal vez esto esté por construirse en este proceso dinámico.

Respecto de las fronteras del me, aunque es claro que incluyen a estudiantes secundarios y universitarios, la implantación del sistema de universidades privadas en nuestro país confiere a estas últimas una situación particular, lo que podría afectar las fronteras de la inclusión de muchas. En cuanto a los oponentes, estos se definen claramente como las autoridades de gobierno con sus políticas en educación y en el plano económico, la banca. Este hecho contribuye teóricamente a su fortaleza, aunque la falta de evidencia respecto de la identificación de otros oponentes asociados al mercado educacional arroja una duda sobre la nitidez de la percepción del alcance del actor contrario.

Además de los aspectos de empoderamiento en el movimiento social señalados anteriormente, es importante la organización, las estrategias y acciones o tácticas llevadas a cabo. Muchos estudios se han centrado en este aspecto de la construcción de la organización, destacando básicamente la horizontalidad (Baeza y Sandoval, 2009; González, 2006; Muñoz, 2002) y participación en la toma de decisiones. Según Muñoz (2002) y González (2006), esto se vincula con su origen en la amistad. Se rechaza el trabajo cupular y en cambio, se opta por una estructura no jerárquica (González 2006; Osorio, 2003). Se incorporan dinámicas muy innovadoras: la asamblea como mecanismo de toma de decisiones (Baeza y Sandoval, 2009; González, 2006) con un estilo de liderazgo estrictamente sujeto a bases, dinámico y cambiante (Baeza y Sandoval, 2009; Osorio, 2003). En este sentido se ejerce una «vocería» de las opiniones de las bases donde se toman las decisiones, aunque las aspiraciones de los líderes puedan ser más trascendentes a las de los demás estudiantes. Su estructura no es rígida y se observa cambio de voceros de acuerdo a decisiones que tome la base, por lo que frente a negociaciones puede existir una multiplicidad de voceros (Baeza y Sandoval, 2009; Osorio, 2003). Su

1 Movimiento de protesta de campesinos de los países bajos, citado en De Weerd \& Klandermans (1999:1073-1095). 
lógica de funcionamiento es de «coordinación» más que de «dirección» (Osorio, 2003). En términos de empoderamiento, esto refleja participación efectiva, toma de decisiones, expresión libre y escucha de las opiniones. Sus líderes tienen una agenda específica y son capaces de explicarla y comunicarla (Baeza y Sandoval, 2009). También tiene importancia la participación individual y débilmente institucionalizada (Baeza y Sandoval, 2009). Asimismo destacan sus protestas bien organizadas y el uso de tecnología virtual en su comunicación en redes (Baeza y Sandoval, 2009; Osorio, 2003). Su organización está basada en la autogestión (Baeza y Sandoval, 2009; Muñoz, 2002; Osorio, 2003) y según Osorio (2003) para no ser controlados y mantener sus formas de proceder. Los aspectos mencionados corresponden a un despliegue de recursos que forma parte del proceso de empoderamiento (Montero, 2003) y que se ha denominado «destrezas interaccionales», que incluyen capacidad de organización, toma de decisiones, entre otros (Zimmerman, 2000).

Las tácticas preferidas son la acción directa (Baeza y Sandoval, 2009; Osorio, 2003), resaltando las protestas, manifestaciones, el «tomarse la calle» y efectuar «funas». ${ }^{2}$ Finalmente, es significativa la acción simbólica para difundir sus mensajes y demandas, representada mediante creaciones de danza, muñecos gigantes ilustrativos de políticos, disfraces, etcétera. Estas creaciones constituyen un espacio de creatividad y expresión. La acción es fundamental en el proceso de empoderamiento, y aquí está presente en una variada gama de formas. Sin embargo, emerge la duda respecto de la identificación masiva con formas más confrontacionales o disruptivas (cortes de calles, barricadas, destrozos de infraestructura de la ciudad, etcétera). Esto forma parte del manejo de diferencias internas, que si no son resueltas, pueden debilitar al ME. Sobre el tema de las diferencias internas antitéticas no hay evidencias que puedan arrojar luz decidora sobre el manejo de dichas controversias.

Finalmente, la fortaleza y empoderamiento experimentado se relaciona con la existencia de oportunidades políticas (circunstancias) externas. En relación a su interacción con el contexto sociopolítico, desde el período de transición democrática, el ME se ha caracterizado por su autonomía respecto de los partidos políticos e institucionalidad gubernamental (Muñoz, 2002; Osorio, 2003) y además, su desconfianza hacia

2 Funa, es una pequeña protesta frente a un lugar físico de vulneración de derechos o frente a un sujeto involucrado en dichas vulneraciones. 
las instituciones (Muñoz, 2002). Se observa un desinterés por los mecanismos tradicionales de hacer política, y en cambio, una vinculación con lo político a partir de su observación de la realidad cotidiana y los sucesos que los afectan o afectan a otros (Baeza y Sandoval, 2009). No les interesa el poder político institucional, sino que su objeto es influir en la opinión pública y en los encargados de tomar decisiones políticas (Osorio, 2003). Si bien los dirigentes de una federación, la Federación de Estudiantes de Chile (FECH), se identifican como de izquierda, muchos no militan en partidos políticos (Carrasco, 2010) y crece la adhesión a nuevas expresiones políticas tales como colectivos y organizaciones de corte asambleísta. Más allá de la relación con la institucionalidad política, poco se sabe sobre eventos que pudieran empoderar o desempoderar al ME. Siguiendo a Meyer y Verduzco (2010), tanto la apertura como la «cerrazón» de las autoridades pueden estimular a un movimiento. En este caso, se puede hipotetizar que en el movimiento del año 2011 está influyendo la cerrazón considerando las afirmaciones juveniles sobre las decisiones gubernamentales que no acogieron las peticiones centrales de los estudiantes en el año 2006 (Ahumada, 2011) y que estimulan la idea de una necesidad ineludible de participar movilizados para generar cambios. A partir del movimiento del año 2011, la historia aún se está escribiendo, por lo que no es claro hasta qué punto las ofertas gubernamentales que no son consideradas satisfactorias, están afectando el curso del movimiento. Finalmente, las oportunidades están «anidadas» en oportunidades internacionales. Desde este punto de vista, el ingreso de Chile a la Organización para la Cooperación y el Desarrollo Económicos (OCDE), con sus parámetros educativos puede haber influido en la legitimación de esta causa y su demanda.

\section{CONCLUSIONES}

En síntesis, las fortalezas del movimiento estudiantil se concentran en torno a la claridad y nivel de acuerdo en sus demandas instrumentales centrales de equidad en la educación mediante una educación pública gratuita y de calidad. Ellas se basan en convicciones profundas de vulneración de derechos sociales de los jóvenes y de amplios sectores de chilenos, debido a la mercantilización de la educación y de otros servicios sociales, privando de acceso a quienes no puedan comprar esos servicios. Esto redunda en un perjuicio para lograr una posición social de calidad en la sociedad y un futuro bienestar subjetivo. 
Ligado a lo anterior, se puede considerar como fortaleza el nivel de cuestionamiento del actual estado de cosas y la existencia de un marco interpretativo global del problema que identifica a una mayoría, no solo de estudiantes, sino que de la población. Este marco contiene ideas de rechazo a la concepción de la educación como bien de consumo y sujeto a negocio con lucro, y levanta las nociones de educación como derecho a ser garantizado por el Estado. La construcción del marco ha avanzado desde el año 2006 a la actualidad, en la que se cuestiona la Constitución Política, el sistema económico y el sistema tributario (Confech, 2011), todos implicados en las posibilidades de efectuar cambios. Sin embargo, el marco no ha consensuado una explicación causal del problema de la mercantilización de la educación, una explicación que tenga en cuenta diferentes niveles y ámbitos (político, económico, cultural, histórico) y que logre aportar un modelo comprensivo global del problema. Aun así, se ve muy clara una solución, que denota pérdida de confianza en el mercado y reclama «más Estado». Pero, ¿es suficiente esto para lograr los cambios? Tendemos a pensar que es necesaria una construcción más elaborada de marco interpretativo para afrontar el marco ideológico hegemónico. Respecto del componente intrapersonal del empoderamiento, se observa una gran motivación de control de parte de los estudiantes. Sin embargo, no es evidente que exista una percepción de lograr influencia política efectiva en relación a sus demandas. Este podría constituir un punto débil del movimiento. Asimismo, no parece fuerte el sentido identitario ligado específicamente al movimiento, lo que podría incidir en una más fácil erosión frente a problemas en el ámbito instrumental. La definición de oponentes es clara y refiere a las autoridades políticas tanto del actual gobierno, como de los gobiernos pasados de la Concertación de Partidos por la Democracia, además de la banca. Sin embargo, esta definición no abarca a otros potenciales oponentes ligados al mercado educacional, lo que limita la percepción del alcance de éstos.

En relación con su nivel de organización, el manejo de tecnologías virtuales, interacción en redes, creatividad y dinamismo constituyen una gran fortaleza y denotan grandes habilidades interaccionales del empoderamiento. También lo son su estilo de participación con influencia de todos en las decisiones, lo que es un hecho empoderador y desarrolla destrezas de liderazgo y/o de sentido de agencia en muchos. La participación directa coincide con las aspiraciones ciudadanas actuales de igualdad política planteadas por Mujica (2010).

En cuanto a las tácticas, permiten desplegar el componente comportamental del empoderamiento de variadas formas. Sin embargo, las 
diferencias respecto de algunas tácticas introducen tensiones, que denotan a su vez algunas diferencias en los marcos interpretativos más amplios de sociedad con los cuales ellos analizan el problema por el que luchan y las vías de solución acordes.

En el empoderamiento, tendríamos dos niveles de contexto, siguiendo el planteamiento de Silva y Martínez (2004): el contexto del movimiento mismo para el empoderamiento a nivel individual de los jóvenes, y el entorno de la institucionalidad política y económica como contexto del nivel colectivo de movimiento. En este caso, las oportunidades políticas refieren al contexto del segundo tipo. Los contextos empoderadores son aquellos que incluyen a las personas en la toma de decisiones (Maton, 2008), y como señalan Rich, Edelstein, Hallman y Wandersman (1995), refieren a la capacidad de las instituciones formales de responder a los ciudadanos e involucrarlos en la toma de decisiones, tener mecanismos de consulta efectivos, ofrecer políticas y/o una legislación que acoge a la comunidad, y responder a las peticiones satisfactoriamente (Rich et al., 1995). Si los mecanismos no existen o no son efectivos, las personas deben hacer grandes esfuerzos, tales como costosas acciones legales y desobediencia civil, para ser oídos (Rich et al., 1995). A la luz de esta conceptualización, el contexto institucional político y económico del movimiento no es empoderador, las ofertas de gobierno han sido rechazadas por el movimiento y las movilizaciones se prolongaron por más de seis meses en el año 2011. Luego del rechazo del movimiento a la oferta efectuada por el Presidente de la República, el Ejecutivo decidió continuar el proceso en el Parlamento, marginando a los estudiantes (Carmona, 2011). Ahora, en el caso de un movimiento social, la falta de apertura efectiva del poder ejecutivo puede ser también motivo de fortalecimiento, ya que se pueden acrecentar otros aspectos, como la definición de oponente, identidad, etcétera, por lo que frente a este punto no hay suficiente información para plantear una conclusión. En este y otros aspectos inciertos, tendremos que esperar el avance del proceso del movimiento estudiantil y desarrollar mayor investigación para dar respuestas.

SANTIAGO (CHILE), ENERO 2013

RECIBIDO: ENERO 2013 ACEPTADO: FEBRERO 2013 


\section{REFERENCIAS BIBLIOGRÁFICAS}

ADIMARK (2011, septiembre): «Encuesta: evaluación gestión del gobierno. Informe mensual».

AHUmADA, R. (2011): «Los errores del 2006 que la Confech no quiere repetir». The Clinic, Santiago, 13 septiembre.

BAEZA J. y M. SALDOVAL (2009): «Valores en estudiantes de educación media de la Región Metropolitana». Santiago: CEJU/UCSH (inédito).

BRONFENBRENNER, URIE y STEPHEN CECI (1994): «Nature-nurture reconceptualized in developmental perspective: A bioecological model». Psychological Review, $\mathrm{N}^{\circ} 101$ (4).

CARMONA, ALEJANDRA (2011): «Los factores que amenazan la proyección del movimiento estudiantil». El Mostrador, 21 noviembre.

CARRASCO, GIOVANI (2010): «Participación y tendencias políticas en estudiantes universitarios: el caso de la Universidad de Chile». Última Década $\mathrm{N}^{\circ} 32$. Valparaíso: Ediciones CIDPA.

CONFEDERACIÓN DE ESTUDIANTES DE CHILE (2011): «Bases técnicas para un sistema público gratuito de educación para Chile». Santiago: CONFECH.

Contreras, PaOla, Osvaldo CoRRales y JuAn SANDOVAL (1996): «Representación social de la pobreza y los pobres en jóvenes de Valparaíso». Proposiciones $\mathrm{N}^{\circ} 27$. Santiago: Ediciones Sur.

DE WEERD, MARGa y BERT Klandermans (1999): «Group identification and political protest: Farmers protest in The Netherlands». European Journal of Social Psychology №29.

FALERO, ALFREDO (2007): «Subjetividad colectiva y movimientos sociales. Una perspectiva para examinar los actuales procesos sociopolíticos y los escenarios posibles en el cono sur». Anais do II Seminário Nacional, Movimentos Sociais, Participação e Democracia. Florianópolis: UFSC.

GoNZÁlEZ, JuAN (2006): «Ciudadanía juvenil en el Chile post dictadura. El movimiento secundario del año 2006 y las organizaciones de autoeducación popular». Disponible en www.opech.cl.

HUIDOBRO, JUAN EDUARDO (2011): «Movilizaciones estudiantiles, un intento de interpretación».

KIRSHNER, BEN (2006): «Power in numbers: The social formation of civic identities in a youth organizing campaign». Paper presentado en Society for Research on Adolescence, San Francisco, CA.

KLANDERMANS, BERT (2003): «Collective political action». En DAVID SEARS, LEONIE HUDDY y ROBERT JERVIS (eds.): Oxford handbook of political psychology. New York: Oxford University Press.

Martínez, María Loreto; CARMen Silva y Ana C. HernándeZ (2010): «¿En qué ciudadanía creen los jóvenes? Creencias, aspiraciones de ciudadanía y motivaciones para la participación sociopolítica en jóvenes». Psykhe $\mathrm{N}^{\circ}$ 19. Santiago: Escuela de Psicología, Pontificia Universidad Católica de Chile. 
- - — MARIELA CARMONA y PATRICIO CUMSILle (2012): «Young chileans views of citizenship: Findings from the first generation born after the reinstitution of democracy». Applied Developmental Science $\mathrm{N}^{\circ} 16(4)$.

MATON, K (2008): «Empowering community settings: Agents of individual development, community betterment, and positive social change». American Journal of Community Psychology $\mathrm{N}^{\circ} 41(1 / 2)$.

MEYER, DAVID y DAISY VERDUZCO (2010): «Social movements and contentious politics». En KEVIN T. LEICHT y J. CRAIG JENKINS (eds.): Handbook of politics. State and society in global perspective. New York, Dordrecht, Heidelberg \& London: Springer.

MONTERO, MARITZA (2003): Teoría y práctica de la psicología social comunitaria. Buenos Aires: Paidós.

MujICA, PEDRo (2010): La igualdad política: el significado actual de la participación ciudadana. Santiago: Ril Editores.

MUÑOZ, VícTOR (2002): «Movimiento social juvenil y eje cultural. Dos contextos de reconstrucción organizativa (1976-1982/1989-2002)». Última Década $\mathrm{N}^{\circ} 17$. Valparaíso: Ediciones CIDPA.

OLSON, MANCUR (1992): La lógica de la acción colectiva, bienes públicos y la teoría de grupos. México: Limusa-Noriega.

OSORIO, CAROLINA (2003): «Las nuevas formas de acción colectiva: nuevos movimientos contestatarios juveniles en Santiago de Chile». Informe final del concurso «Movimientos sociales y nuevos conflictos en América Latina y el Caribe». Programa Regional de Becas CLACSO.

PINQUART, MARTIN y RAINER SILBEREISEN (2004): «Human development in times of social change: Theoretical considerations and research needs». International Journal of Behavioral Develpment $\mathrm{N}^{\circ} 28(4)$.

RAPPAPORT, JULIAN (1981): «In praise of paradox: A social policy of empowerment over prevention». American Journal of Community Psychology $\mathrm{N}^{\circ} 9$.

Rich, RichaRd, Michael EDELTEIN, William HaLlman y ABRAHAM WANDERSMAN (1995): «Citizen participation and empowerment: The case of local environmental hazards». American Journal of Community Psychology $\mathrm{N}^{\circ} 23(5)$.

SALAZAR, GABRIEL (2011): «El movimiento estudiantil no ha perdido masividad». Radio Cooperativa, Santiago, 7 noviembre.

SILVA, C. y G. MARTínEZ (2004): «Empoderamiento: proceso, nivel y contexto». Psykhe $\mathrm{N}^{\circ}$ 13(2). Santiago: Escuela de Psicología, Pontificia Universidad Católica de Chile.

SILVA, EdUARDo y CARMEN SILVA (2010): «La economía política y las motivaciones de participación sociopolítica de jóvenes chilenos: una interpretación de los hallazgos de Martínez, Silva y Hernández». Psykhe No19(2). Santiago: Escuela de Psicología, Pontificia Universidad Católica de Chile. 
Simon, B.; M. Loewy, M. StÜRmer, U. Webero P. Freytag, C. Habig, C. KAMPMEIER y P. SPAHLINGER (1998): «Collective identification and social movement participation». Journal of Personality and Social Psychology $\mathrm{N}^{\circ} 74(3)$.

ZIMMERMAN, MARC (2000): «Empowerment theory». En JULIAN RAPPAPORT y EDWARD SEIDMAN (eds.): Handbook of Community Psychology. New York: Kluwer. 